\title{
Anomalous Phase and Attenuation Constants of SiC Rod Waveguide at Higher Temperatures
}

\author{
T. GRIC AND L. NiCKELSON \\ ${ }^{a}$ State Research Institute Center for Physical Sciences and Technology, Vilnius, LT-01108, Lithuania \\ ${ }^{b}$ Vilnius Gediminas Technical University, Vilnius, LT-10223, Lithuania
}

(Received August 24, 2012; in final form November 10, 2012)

Here we present the dependences of the phase and attenuation constants of silicon carbide ( $\mathrm{SiC}$ ) cylindrical rod waveguide upon frequency at different temperatures, i.e. $T=1800^{\circ} \mathrm{C}$ and $T=1900{ }^{\circ} \mathrm{C}$ when the rod radius is relatively large. We have also calculated the electric and magnetic field distributions at different frequencies in order to explain the anomalous attenuation. The $\mathrm{SiC}$ rod waveguide was investigated by the partial area method. The complex roots have been found by the Müller method. Computer codes for calculations were written in MATLAB language.

DOI: $10.12693 /$ APhysPolA.123.58

PACS: 42.68.Ay, 84.40.Az, 81.05.Je, 03.50.De

\section{Introduction}

Silicon carbide $(\mathrm{SiC})$ has revolutionised semiconductor power device performance. It is a wide band gap semiconductor with an energy gap wider than $2 \mathrm{eV}$ and possesses extremely high power, high voltage switching characteristics and high thermal, chemical and mechanical stability [1].

The $\mathrm{SiC}$ waveguides have a wide range of applications. Hydrogenated amorphous silicon carbide optical waveguides for telecommunication wavelength applications is proposed and demonstrated in [2]. The work [3] presents a simple evanescent wave-sensing system based on plasma-enhanced chemical vapour deposition silicon carbide waveguides.

Engineers have been investigating and used circular cylindrical waveguides for many years due to their excellent electrodynamical characteristics (e.g. a large broadband width) and the wide possibilities for their implementation in microwave and optoelectronic devices as well as solid-state electronics. Dispersion characteristics for the low-loss waveguide modes, the linearly-polarized $\mathrm{HE}_{11}$ mode and the $\mathrm{TE}_{01}$ mode are obtained in [4]. The experimental results are compared to the metallic waveguide dispersion.

Thus flexible dielectric waveguides have been demonstrated at $10 \mathrm{GHz}$ and $94 \mathrm{GHz}$ in [5] using thin-wall polymer tubing filled with low-loss, high-dielectric-constant powders. We can see in Figs. 3-5 of article [5] that usual dispersion characteristics of dielectric rod waveguide for the main (fundamental) and higher modes are smooth curves.

Nowadays a large stream of articles is dedicated to the investigations of the anomalous group-velocity, phase and attenuation constants in order to reach some very new unconvincing properties. The first experimental demonstration of anomalous group-velocity dispersion in silicon waveguides across the telecommunication bands is presented in [6]. The dispersive and anomalous refraction properties of one- and two-dimensional photonic crystal waveguides are explained both phenomenologically and by simulations in [7]. Possible new ways for applications of semiconductor photonic bandgap devices are also discussed in this work. It is shown in [8] that modes of axially uniform waveguides can be made to have anomalous dispersion relations resulting from strong repulsion between two modes. From the literature review we see that the topic of the anomalous dispersion is actual and is widely investigated.

In this article we present the investigations of the anomalous dispersion in the absorptive circular silicon carbide waveguides at two higher temperatures and two radii.

\section{The partial area method}

The dispersion equation for the waveguide under our consideration is as follows [9]:

$$
\begin{aligned}
& \psi^{2} \xi^{2} h^{2} m^{2}\left(\frac{1}{\sigma r}-\frac{1}{\varsigma r}\right)^{2}+\left(\psi \psi^{\prime} \xi \xi^{\prime}\right) \\
& \times \frac{k^{2}}{\sqrt{\varsigma} \sqrt{\sigma}}\left(\varepsilon_{\mathrm{r}}^{\mathrm{SiC}} \mu_{\mathrm{r}}^{\mathrm{a}}+\varepsilon_{\mathrm{r}}^{\mathrm{a}} \mu_{\mathrm{r}}^{\mathrm{SiC}}\right) \\
& -\psi\left(\xi^{\prime}\right)^{2} \frac{\varepsilon_{\mathrm{r}}^{\mathrm{SiC}} \mu_{\mathrm{r}}^{\mathrm{SiC}} k^{2}}{\sigma}-\left(\psi^{\prime}\right)^{2} \xi^{2} \frac{\varepsilon_{\mathrm{r}}^{\mathrm{a}} \mu_{\mathrm{r}}^{\mathrm{a}} k^{2}}{\varsigma}=0,
\end{aligned}
$$

where $\psi=J_{m}\left(k_{\perp}^{\mathrm{a}} r\right), \xi=H_{m}\left(k_{\perp}^{\mathrm{SiC}} r\right), \psi^{\prime}$ and $\xi^{\prime}$ are derivatives of magnitudes $\psi$ and $\xi$, correspondingly, $\varepsilon_{\mathrm{r}}^{\mathrm{SiC}}$ and $\varepsilon_{\mathrm{r}}^{\mathrm{a}}$ are the permittivity of the $\mathrm{SiC}$ and the air medium, correspondingly, $\mu_{\mathrm{r}}^{\mathrm{SiC}}$ and $\mu_{\mathrm{r}}^{\mathrm{a}}$ are the permeability of the $\mathrm{SiC}$ and the air medium, correspondingly, $\sigma=\left(k_{\perp}^{\mathrm{SiC}}\right)^{2}, \varsigma=\left(k_{\perp}^{\mathrm{a}}\right)^{2}$. Magnitudes $k_{\perp}^{\mathrm{SiC}}$ and $k_{\perp}^{\mathrm{a}}$ are related with the longitudinal propagation constant and the permittivity and permeability of the $\mathrm{SiC}$ or the air medium as follows: $k_{\perp}^{\mathrm{SiC}}=\left(k^{2} \varepsilon_{\mathrm{r}}^{\mathrm{SiC}} \mu_{\mathrm{r}}^{\mathrm{SiC}}-h^{2}\right)^{1 / 2}$, $k_{\perp}^{\mathrm{a}}=\left(k^{2} \varepsilon_{\mathrm{r}}^{\mathrm{a}} \mu_{\mathrm{r}}^{\mathrm{a}}-h^{2}\right)^{1 / 2}$, where $k=\omega / c$ is the wave number in the air medium, $h$ is the complex longitudinal propagation constant. 


\section{The investigation of the $\mathrm{SiC}$ waveguide}

The object under investigation is the open (without metal screen) circular $\mathrm{SiC}$ waveguide with relatively large radii $2.2 \mathrm{~mm}$ and $3.2 \mathrm{~mm}$ in the operating frequency range $5-70 \mathrm{GHz}$ at higher temperatures $T=1800^{\circ} \mathrm{C}$ and $T=1900^{\circ} \mathrm{C}$. The permittivity of this material is $11-7 \mathrm{i}$, when $T=1800^{\circ} \mathrm{C}$ and $12-8 \mathrm{i}$, when $T=1900^{\circ} \mathrm{C}$ at the cutoff frequencies of the main modes [10]. It is known that the imaginary part of the permittivity is dependent upon frequency like

$$
\operatorname{Im}\left(\varepsilon_{\mathrm{r}}\right)=\frac{1}{\omega \varepsilon_{0} \rho},
$$

where the value $\rho$ is the semiconductor $\mathrm{SiC}$ material specific resistivity, $\omega$ is the cyclic frequency, $\varepsilon_{0}$ is the dielectric constant.

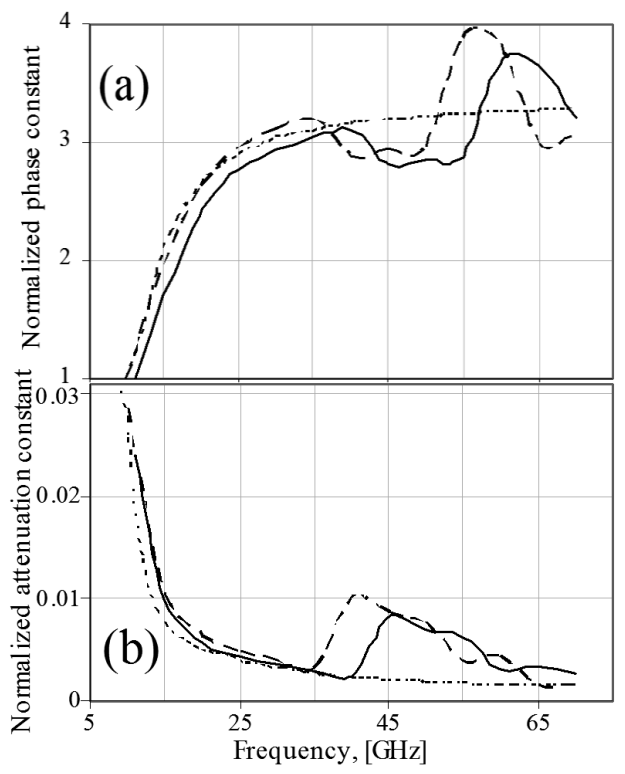

Fig. 1. The dispersion characteristics of the $\mathrm{SiC}$ waveguide. When $T=1800{ }^{\circ} \mathrm{C}$ and $r=2.2 \mathrm{~mm}$, the main mode is denoted by the solid line, when $T=1900^{\circ} \mathrm{C}$ and $r=2.2 \mathrm{~mm}$ - by the dashed line, when $T=1800^{\circ} \mathrm{C}$ and $r=2.5 \mathrm{~mm}$ - by the dotted line [11].

In Figs. 1 and 2 we see the dispersion characteristics of the investigated waveguide, when the radius of the waveguide is $r=2.2 \mathrm{~mm}, r=2.5 \mathrm{~mm}$ and $r=3.2 \mathrm{~mm}$. In Figs. 1, 2a we present the dependence of the real part of longitudinal propagation constant upon frequency, in Figs. 1, 2b - the dependence of the imaginary part of longitudinal propagation constant upon frequency. Here we present the dispersion characteristics only of the main modes propagating in the $\mathrm{SiC}$ waveguide. In Figs. 1 and 2 real (a) and imaginary (b) parts are shown of the complex longitudinal propagation constant $h=h^{\prime}-h^{\prime \prime} \mathrm{i}$, which are normalized by the wave number $k, h^{\prime}$ is the phase constant, $h^{\prime \prime}$ is the attenuation constant.

In Figs. 1, 2 we see that there are inhomogeneous values of $h^{\prime} / k$ and $h^{\prime \prime} / k$ on the frequency. We see that real

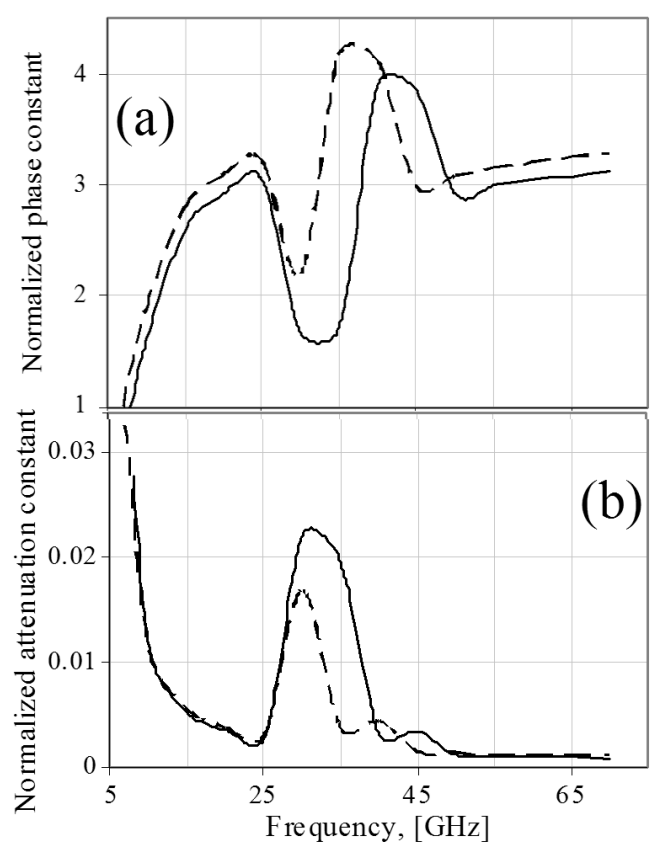

Fig. 2. The dispersion characteristics of the $\mathrm{SiC}$ waveguide, when $r=3.2 \mathrm{~mm}$. When $T=1800^{\circ} \mathrm{C}$, the main mode is denoted by the solid line, when $T=1900^{\circ} \mathrm{C}-$ by the dashed line.

and imaginary parts of dispersion characteristics have unusual protrusions with minimal and maximal values in the narrow frequency area. The last essential attribute can be used for creation of sensitive modulators, filters, absorbers and so on. In Figs. 1, 2 we see that when the radius increases the points of minimum and maximum move to the lower frequency range. The dependence of position on the frequency axis of protrusion extrema is an intricate one and should be considered for each specific parameter, i.e. radius, $\mathrm{SiC}$ permittivity.

In Figs. 1, 2a we see that when the radius of the waveguide and the temperature decreases the cutoff frequency of the main mode moves to the higher frequency range. In Figs. 1, 2a we see that when the temperature and the radius of the waveguide increase, the value of the phase constant peak increases. Analyzing the characteristics depicted in Figs. 1, 2b, we see that dependence of the waveguide losses upon temperature is quite complicated.

The values of the losses could change when the temperature increases. However this process depends upon the waveguide radius.

We could mention that when the radius increases the losses increase. The appearance of the points of minimum and maximum in Figs. 1 and 2 could be explained as follows. The electromagnetic field concentrates near the waveguide boundary, i.e. at the junction of the air and $\mathrm{SiC}$ environments. The interaction between the $\mathrm{SiC}$ and air acts as the concentrator of electromagnetic field of the waveguide main mode. Changing the frequency we could achieve the case when the field is drawn into or pushed out the waveguide. 
It needs to be mentioned that we have already calculated the dispersion characteristics of the circular waveguide made of $\mathrm{SiC}$ material, when $r=2.5 \mathrm{~mm}, T=$ $1800{ }^{\circ} \mathrm{C}$ in [11]. We should state that the real and imaginary parts of dispersion characteristics do not have unusual protrusions in this case.

We have calculated the electric and magnetic field distributions of the main mode propagating in the circular $\mathrm{SiC}$ waveguide with radius $r=3.2 \mathrm{~mm}$. In Fig. 3 we present the electric field distribution when frequency is $f=25 \mathrm{GHz}$. The unusual protrusion of attenuation constant has a low value (see Fig. $2 \mathrm{~b}$ ) at $f=25 \mathrm{GHz}$.

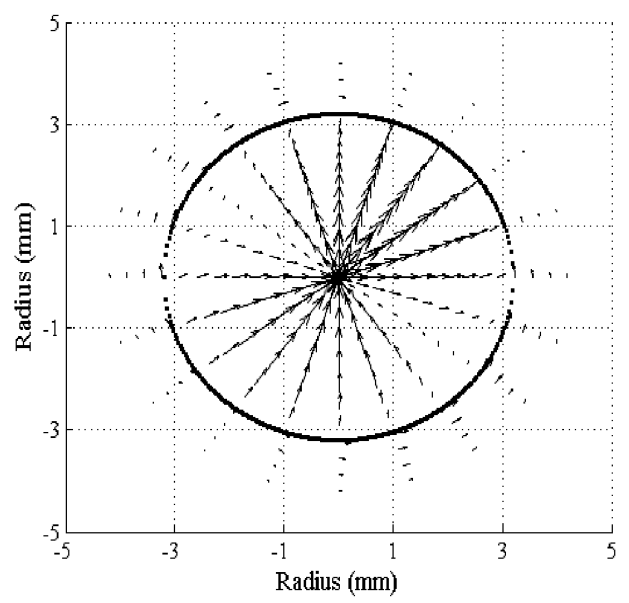

Fig. 3. The electric field distribution of the main mode when radius of the waveguide is $r=3.2 \mathrm{~mm}, f=$ $25 \mathrm{GHz}$.

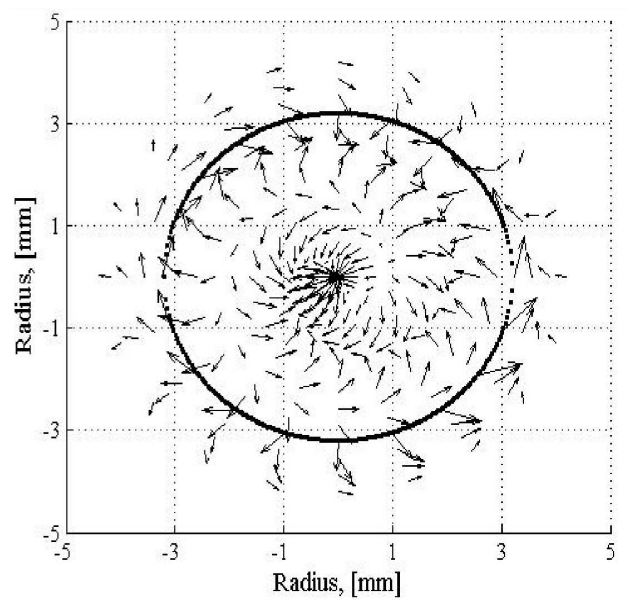

Fig. 4. The electric field distribution of the main mode when radius of the waveguide is $r=3.2 \mathrm{~mm}, f=$ $30 \mathrm{GHz}$.

In Fig. 4 we present the electric field distribution when frequency is $f=30 \mathrm{GHz}$. The unusual protrusion of attenuation constant has a high value (see Fig. $2 b$ ) at $f=30 \mathrm{GHz}$.

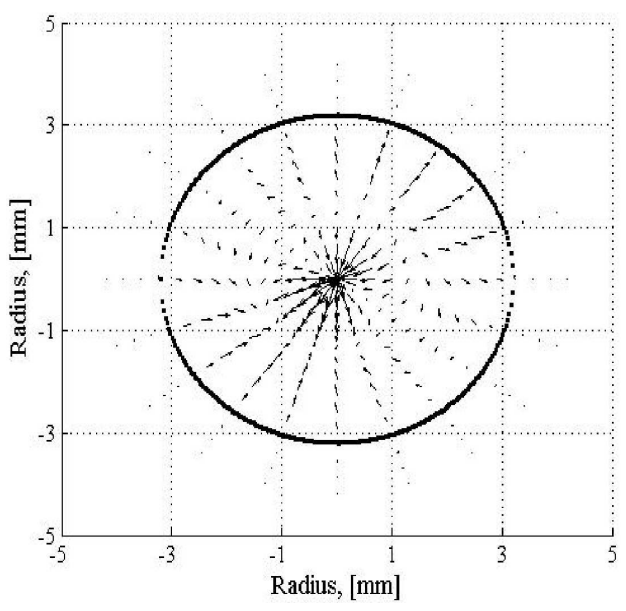

Fig. 5. The electric field distribution of the main mode when radius of the waveguide is $r=3.2 \mathrm{~mm}, f=$ $70 \mathrm{GHz}$.

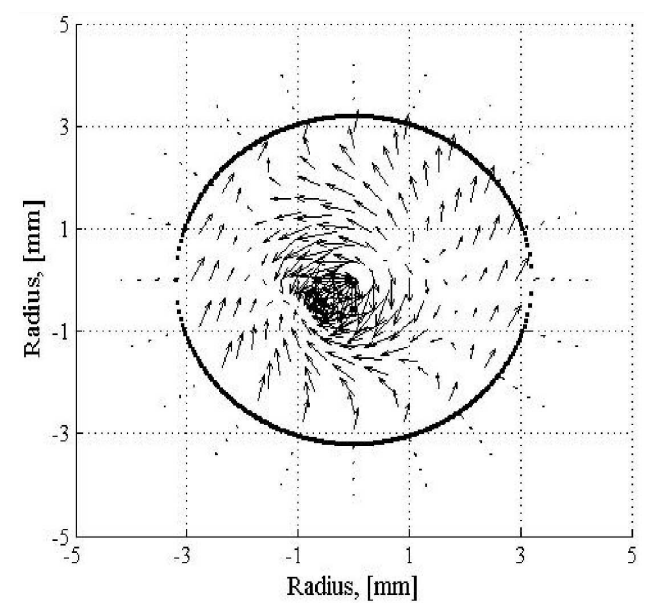

Fig. 6. The magnetic field distribution of the main mode when radius of the waveguide is $r=3.2 \mathrm{~mm}$, $f=25 \mathrm{GHz}$.

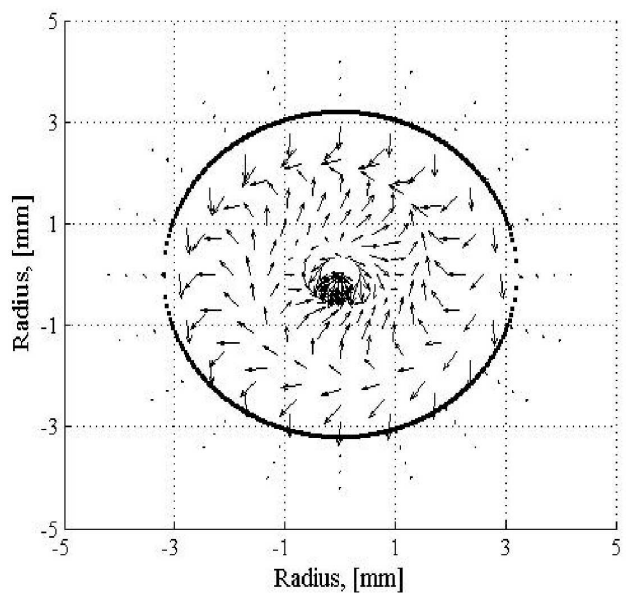

Fig. 7. The magnetic field distribution of the main mode when radius of the waveguide is $r=3.2 \mathrm{~mm}$, $f=30 \mathrm{GHz}$. 


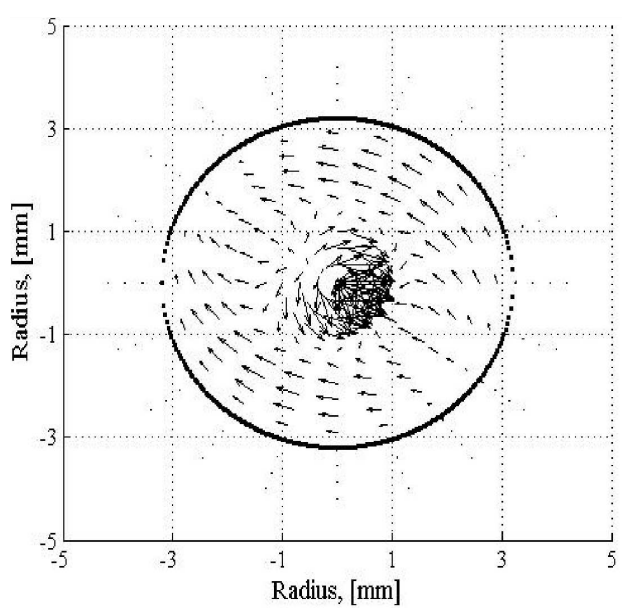

Fig. 8. The magnetic field distribution of the main mode when radius of the waveguide is $r=3.2 \mathrm{~mm}$, $f=70 \mathrm{GHz}$.

In Fig. 5 we present the electric field distribution when frequency is $f=70 \mathrm{GHz}$.

In Figs. 6-8 we present the magnetic field distributions when frequency is $f=25 \mathrm{GHz}, f=30 \mathrm{GHz}$ and $f=$ $70 \mathrm{GHz}$, correspondingly.

We see that when the frequency increases the imaginary part of the permittivity of $\mathrm{SiC}$ decreases (see formula (2)). In spite of this the dispersion curve has the anomalous peak of protrusion at frequency about $30 \mathrm{GHz}$ when $r=3.2 \mathrm{~mm}$. Comparing Figs. $3-5$ we see that the electric field distributions at $25 \mathrm{GHz}$ and $70 \mathrm{GHz}$ are similar and the electric field distribution at $30 \mathrm{GHz}$ differs from them. The components of the electric field are large and have tangential behavior on the waveguide surface at $30 \mathrm{GHz}$ (see Fig. 4). The large waveguide losses at the last frequency are caused by the structure of electric field which concentrates in $\mathrm{SiC}$ material in special way. The magnetic field strength lines are closed and have different distributions at every frequency (Figs. 6-8).

\section{Conclusions}

- We have created the new algorithm for investigation of the waveguides made of absorptive materials.

- We have discovered the anomalous behaviour of the dispersion characteristics at the certain waveguide radius and the frequency range.
- When the waveguide radius increases the extreme points of unusual protrusion of attenuation and phase constants move to the lower frequency range.

- When the waveguide radius increases the maximal value of attenuation constant protrusion increases.

- The extrema points of the attenuation constant move to the higher frequency range when the temperature increases.

\section{References}

[1] M. Vashishath, A.K. Chatterjee, Mj. Int. J. Sci. Tech. 2, 444 (2008).

[2] S. Yuya, N. Koichi, S. Youichi, K. Kenji, K. Hitoshi, M. Masahiko, K. Toshihiro, Appl. Phys. Expr. 3, 122201 (2010)

[3] G. Pandraud, P.J. French, P.M. Sarro, in: Proc. IEEE Sensors 2007 Conf., Ed. H.T. Nagle, IEEE, Stoughton (WI) 2007, p. 395 .

[4] O. Mitrofanov, J.A. Harrington, Opt. Expr. 18, 1898 (2010).

[5] W.M. Bruno, W.B. Bridges, IEEE Trans. Microwave Theory Techn. 36, 882 (1988)

[6] A.C. Turner, C. Manolatou, B.S. Schmidt, M. Lipson, M.A. Foster, J.E. Sharping, A.L. Gaeta, Opt. Expr. 14, 4357 (2006).

[7] R. Zengerle, Adv. Solid State Phys. 44, 105 (2004)

[8] M. Ibanescu, S.G. Johnson, D. Roundy, C. Luo, Y. Fink, J.D. Joannopoulos, Phys. Rev. Lett. 92, 063903 (2004)

[9] L. Nickelson, T. Gric, S. Asmontas, in: Properties and Applications of Silicon Carbide, Ed. G. Rosario, InTech, India 2011, p. 535.

[10] T.A. Baeraky, Egypt. J. Solids 25, 263 (2002).

[11] L. Nickelson, T. Gric, S. Asmontas, R. Martavicius, Electron. Electr. Eng. 93, 87 (2009). 